Original Research Paper

\title{
Distributed and Parallel Decision Forest for Human Activities Prediction: Experimental Analysis on HAR-Smartphones Dataset
}

\author{
${ }^{1}$ Budi Padmaja, ${ }^{2}$ Venkata Rama Prasad Vaddella and ${ }^{3}$ Kota Venkata Naga Sunitha \\ ${ }^{1}$ Department of Computer Science and Engineering, Institute of Aeronautical Engineering, Hyderabad, Telangana, India \\ ${ }^{2}$ Department of Computer Science and Engineering, Sree Vidyanikethan Engg College, Tirupati, andhra Pradesh, India \\ ${ }^{3}$ Department of Computer Science and Engineering, BVRITH College of Engg for Women, Hyderabad, Telangana, India
}

Article history

Received: 13-02-2019

Revised: 30-03-2019

Accepted: 10-05-2019

Corresponding Author:

B. Padmaja

Deprtment of Computer

Science and Engineering,

Institute of Aeronautical

Engineering, Hyderabad,

Telangana, India

Email: b.padmaja@gmail.com

\begin{abstract}
Sensor-based human motion detection requires the subtle amount of knowledge about various human activities from fitted sensor observations and readings. The prevalent pattern recognition methodologies have made immense progress over recent years. Nonetheless, these kind of methods usually rely on the particular heuristic variable extraction, which could inhibit generalization realization. This paper presents a distributed and parallel decision forest approach for modeling the Human Activity Recognition Using Smartphones Data. We made an attempt to achieve an optimal generalization performance with possible reduction in overfitting. Later, we compared the performance of proposed procedure with some existing approaches. It is observed that our adopted procedure outperforms with comparatively better statistical performance measures. It also gained $4.7 \mathrm{x}$ speed up in computation.
\end{abstract}

Keywords: Smart Environments, Parallel Processing, Deep Learning, Human Action, Random Oblique KNN, Dual Problem, Decision Forest

\section{Introduction}

Human activity and motion recognition has a significant stint usually in people's routine life. This domain is somewhat related as pattern matching scenario. Traditional pattern detection methods made good amount of progress on human motion recognition field (Bhattacharya and Lane, 2016; Chen et al., 2016). Figure 1 presents an outlook for various example positions of human motion and its modeling through machine learning.
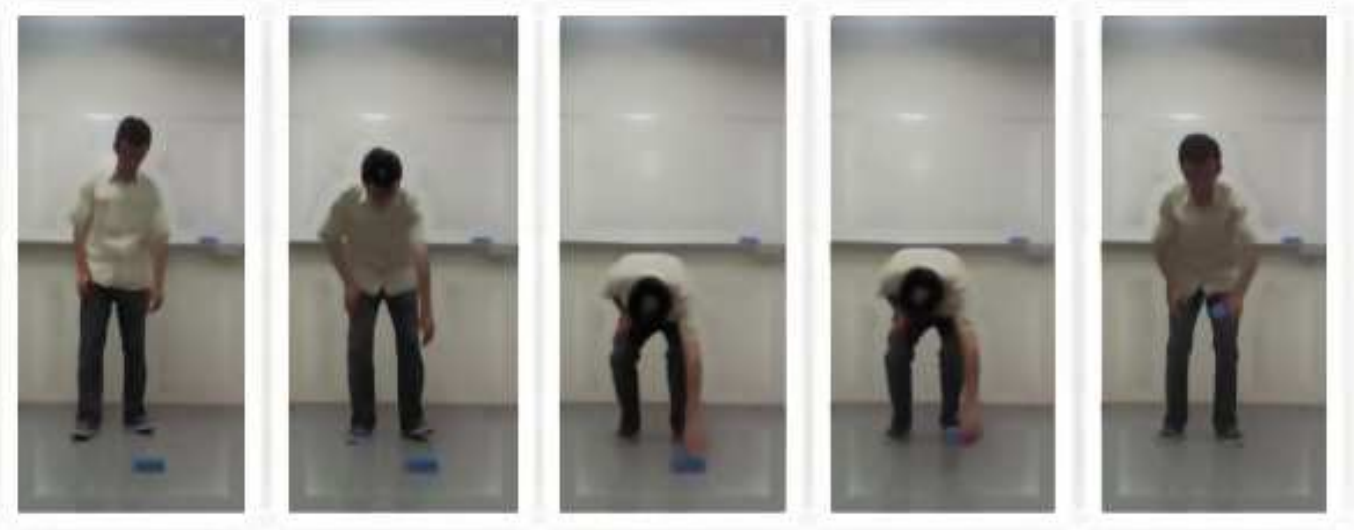

Fig. 1: Various human actions 
Recent past decade witnessed the agile expansion and advancement of various learning routines (Cheng and Scotland, 2017; Ha and Choi, 2016; Ha et al., 2015), which accomplishes unprecedented enforcement in plentiful areas e.g., visual object cognizance, natural language processing, inference reasoning and so on.

\section{Purpose of Study and Contribution Highlights}

Video frames based human activities predictive analysis and motion analysis on sensor (accelerometer, bluetooth, gyroscope devices etc.) captured data are generally two flavours of this domain. This paper primarily focus on sensor-based HAR. As our contribution:

- This paper presents a distributed and parallel decision forest approach for medeling the Human Activity Recognition Using Smartphones Data. We made an attempt to achieve an optimal generalization performance with possible reduction in overfitting

- The proposed model architecture reflects the computational benefits such as - (i) more relaxed classification error bound (ii) speed-up through parallel architecture (iii) reduction in the size of resultant dual problem

- We also compared the empirical performance of proposed procedure with some existing approaches

\section{Literature Review}

This section inspects some significant literature in this domain. Anguita et al. $(2012 ; 2013)$ gave a system prototype for physical activity recognition utilizing smartphone embedded sensors. Same authors, in 2013, described a dataset which made available publically for human motion predictive analysis employing smartphones. Jindong Wang et al. (2017) presented a systematic review and associated challenges on Deep Learning impacts for sensor-based activity perception. Almaslukh et al. (2017), in their work, utilized DL methodology - named Stacked Autoencoder (SAE) to boost the recognition certainty, accuracy and decrease the prediction time. Bhattacharya and Lane (2016) carried out a smartwatch centric probe of activity detection under well known deep learning procedure Restricted Boltzmann Machines. Chen et al. (2016); Padmaja et al. (2018b) enforced LSTM networks for the object mobility recognition. Cheng and Scotland (2017) constructed a Activity Recognition model using Deep Neural Nets. M. Edel and Kppe (2016) given a binarized RNN whose weight parameters, input and the intermediate hidden layer output signals, are all binaryvalued and they need only the basic bit inference for the computational evaluation and the training formation.
Gjoreski et al. (2016) analyzed the deep learning and the classical ML procedures on the object activity detection using the wrist accelerometer. Ha and Choi (2016) given CNNs extended architectures, especially the CNN-pff structures, for the multi-modal statistical data. We utilize both the partial weight sharing sub procedure and the full weight sharing for models in such a way that the modality-specific peculiarity as well as common trait across modalities are being learned from multi-sensor data and are ultimately being aggregated in the upper layers. Same authors later in 2015, adapted the multi-sensor convolutional neural networks for the specific activity recognition (Ha et al., 2015). Hannink et al. (2017) gave a procedure to explicate the abstract information provisioned by wearable sensors to the context-pertinent expert attributes based on the deep convolutional nets. $\mathrm{Hu}$ et al. (2016) suggested SSARTMAP-AR, which is a self-supervised cumulative learning AR model which is updated from $\mathrm{Wi}-\mathrm{Fi}$, Bluetooth, GPS etc. without users elucidation effort. Padmaja et al. (2016a; Sunitha et al., 2018; Padmaja et al., 2016b; 2016c; 2018c) given approaches, for activity recognition in various environments like WSNs, IoT etc. and analyzes the challenges involved in application to practical scenarios. The drawbacks in existing system prototypes are analyzed through an extensive review here, which drove the development of the proposed model.

\section{Organization of the Paper}

Remaining content of the paper is structured as Some significant preliminaries and definitions are given in section 2. The proposed framework is given in section 3. Our empirical experimental evaluation and analysis is presented in section 4. Finally, the paper concludes with section 5 .

\section{Significant Preliminaries, Definitions}

\section{Machine Learning}

Machine learning is a process where a system is artificially educated to perceive the designs by giving it information and a calculation which helps in understanding that information. We allude this procedure of learning as 'preparing' and the yield of this procedure is alluded as a 'demonstrate'. The model is encouraged with new information (or test information) and it can reason about this new data dependent on what it has recently realized. Machine learning models decide a lot of guidelines utilizing immense measures of figuring power that a human mind would be unequipped for preparing. The more information a machine learning model is encouraged, the more unpredictable the principles and the more exact the expectations will be. To condense, the objective of machine learning is to comprehend the structure of information with the goal 
that exact expectations can be made dependent on the properties of that information. While a factual model is probably going to have a natural rationale that can be comprehended by many people, the rules derived by machine learning are regularly outside human ability to grasp on the grounds that our minds are unequipped for processing and breaking down gigantic informational collections.

\section{Supervised Learning}

Deals with make the function learn from the available training part of dataset. A supervised learning procedure exploits the available training data part and makes an inferred function, which can be then exploited further for mapping new ones. Multiple supervised learning algorithms are available such as Support Vector Machines, Neural Networks and Naïve Bayes classifiers etc.

\section{Unsupervised Learning}

Deals with unlabelled data without taking any previously-defined dataset for model training. Unsupervised learning can be thought as a potent tool for look for patterns and trends and analyzing available data. There are various approaches used by unsupervised learning e.g., hierarchical clustering, Kmeans clustering, self-organizing maps etc. Numerous significant learning types are:
1) Active learning

2) Kernel-based learning

3) Transfer learning

4) Distributed learning

5) Association rule learning

6) Inductive logic programming

7) Reinforcement learning

8) Similarity and metric learning

- Definition 2.1.3.1 Consider, a domain $D$ which is a two-tuple $(\chi, P(X)) . \chi$ is taken as the feature space of $D$ and $P(X)$ be the marginal partitioning, where $X$ $=\left\{x_{1} \cdots x_{n}\right\} \in \chi$

- Definition 2.1.3.2 A task $T$ is defined as a two-tuple $(Y, f())$ for some provided domain $D . Y$ is called as the label space of $D$ and $f()$ is an objective predictive function for $D . f()$ is sometimes demonstrated as a conditional probability distribution $P(y \mid x) . f()$ is not given, however can be learned from the training data

\section{Adopted Procedure}

This section presents the adopted framework along with the detailed procedural steps as shown in Fig. 2. Algorithmic procedure 3.1 .1 consists of mainly six components i.e., data admission, data summary, preprocessing, data splitting, model training and predicting generalization performance. Here inside component 5, procedure 3.1.2 is utilized.

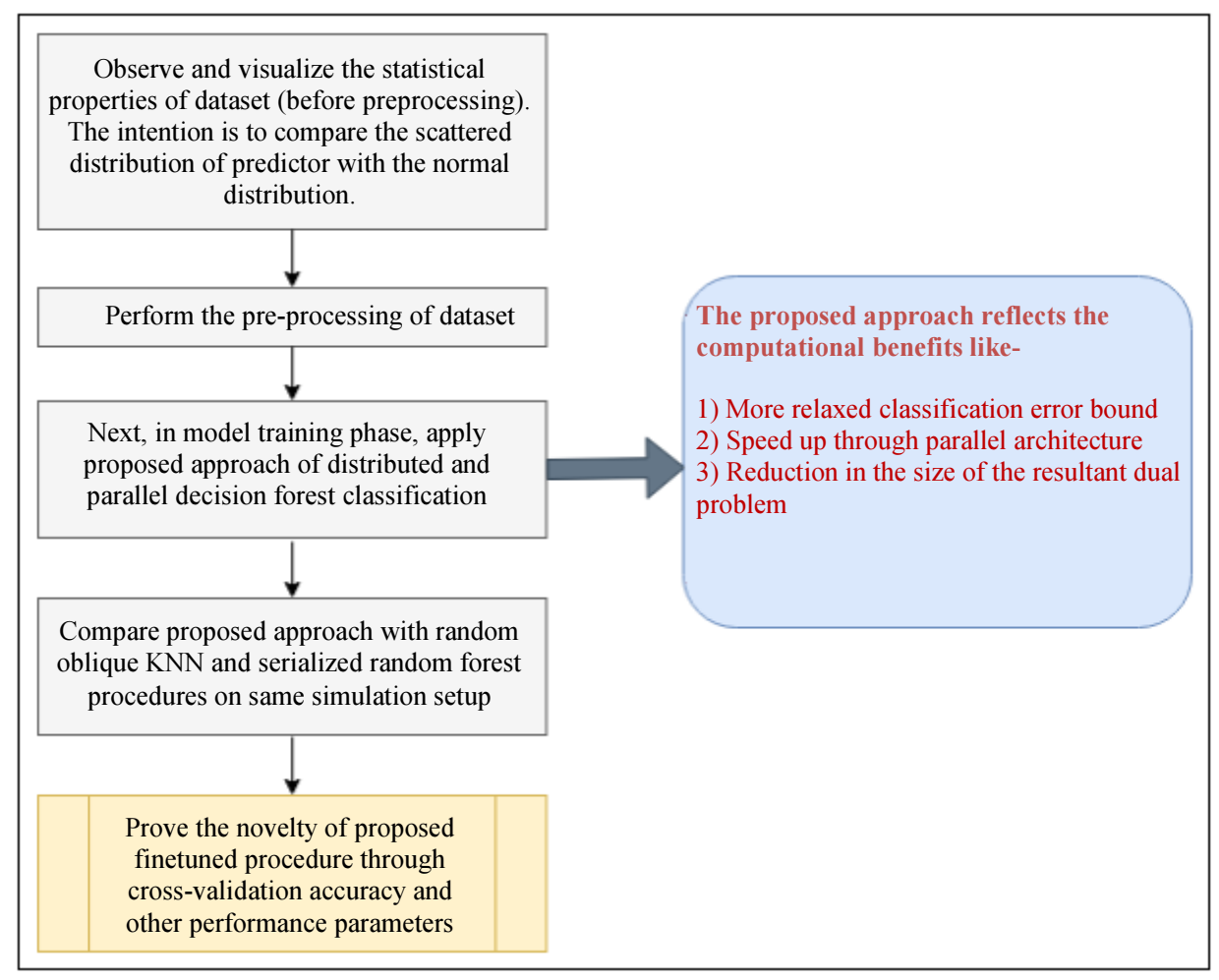

Fig. 2: The framework 


\section{Framework and Algorithmic Steps}

Algorithmic Procedure 3.1.1

1. Data Admission

- Take data as input for processing

2. Data Summary

- Observe and visualize the statistical properties of dataset. The intention for this task is to compare the scattered Distribution of every predictor with the normal distribution

- Observe the distribution of samples with respect to experimental subjects and types of activity

3. Preprocessing

- Perform variable(feature) scaling using Zscaling method

- Perform Near Zero Variance Predictor Detection

- List $_{[H C P]}$ search highly correlated predictors

- $\quad$ Remove $\left(\right.$ list $\left._{[H C P]}\right)$

4. Data Splitting

- Perform cross-validation over every experimental subject and generate different sets of training sample

- Further, split into various CV ensembles for further distributed and parallel processing

5. Model Training

1) Nnodes $\leftarrow \frac{\text { detectcores }}{2}$

2) Setup parallel processing environment by calling the function makeForkCluster()

3) for $t=1$ to $\mathrm{DF}$

- Randomly draw a bootstrap segment ensemble having size as $N$ from training set

- Grow data forest $T_{t}$ to bootstrapped data

- Assign each $\left\{T_{t}\right\}_{1}^{D F}$ to each distributed cores for further computational assignments

- Repeat steps 4 to 6 as recursively for each node of tree structure, until $n_{m}$ is reached (where $n_{m} \rightarrow$ min node size)

4) Choose $x$ variables randomly out of $P$ variables

5) Use procedure 3.1.2 to pick the optimal set of best split-points among $x$

6) Perform the node splitting into $C$ possible choices for each element in the optimal set

7) Output: tree ensembles $\left\{T_{t}\right\}_{1}^{D F}$

8) At a new datapoint $d$, make prediction as: Let, $\hat{L}_{t}(d)$ be class/label prediction of the $t^{\text {th }}$ randomly distributed forest, then - $\hat{L}_{r f}^{D F}(d)=$ majority voterules $\left\{\hat{L}_{t}(d)\right\}_{1}^{D F}$

6. Predicting generalization performance

- Measure how accurately the model is able to predict outcome values for previously unseen data

Procedure 3.1.2

$\operatorname{Proc}\left(A, f_{\text {dis }}\right)$

$A$ : The set of variables(conditional)

$f_{\text {dis: }}$ : Discernibility function

1) $\mathrm{X} \leftarrow \Phi$; besta $=0$;

2) while ( $f_{\text {dis }}$ is non-empty)

3) $\forall v \in A$ that exists in $f_{d i s}$

4) $a=$ heur(v)

5) if(a $>$ besta)

6) besta $=\mathrm{a}$; best_Var $\leftarrow v$

7) $\mathrm{X} \leftarrow X \cup v$

8) $f_{\text {dis }} \leftarrow \operatorname{removeitems}\left(f_{\text {dis }}, v\right)$

9) return $X$

\section{Experimental Results Discussion}

This section describes setup and simulation environment used, dataset description, obtained results and comparisons.

\section{Setup and Simulation Environment}

Our experimental setup contains system environment with $32 \mathrm{~Gb}$ RAM, 5 teraflops computing power. We made two clusters with two CPU's, each with 12 cores (total: $12 \times 2=24 \mathrm{CPU}$ cores). We utilized two nos. of Nvidia GPU's accelerators. We used $\mathrm{R}$ language (v'3.5.1) with R-Studio platform (v'1.1.463).

\section{Packages/Libraries Used}

The various packages/libraries used in the experimental process are - "Matrix", "ggplot2", "plyr", "reshape", "reshape2", "caret", "Parallel", "doparallel", "doMC" and "gmp".

\section{Dataset Description}

The

dataset

used

(https://archive.ics.uci.edu/ml/datasets/human+activity $+\mathrm{r}$ ecognition+using+smartphones; Anguita et al., 2013) in our experimental process is having following characteristics: Number of Instances $=10299$, Number of Attributes $=561$, there were no missing values present, total number of classes/activities - (WALKING, WALKING UPSTAIRS, WALKING DOWNSTAIRS, SITTING, STANDING, LAYING). Using embedded accelerometer and gyroscope, 3-axial linear acceleration 
and 3-axial angular velocity at a constant rate of $50 \mathrm{~Hz}$ is captured. The experiments have been videorecorded to label the data manually. The obtained dataset has been randomly partitioned into two sets, where $70 \%$ of the volunteers was selected for generating the training data and $30 \%$ the test data.

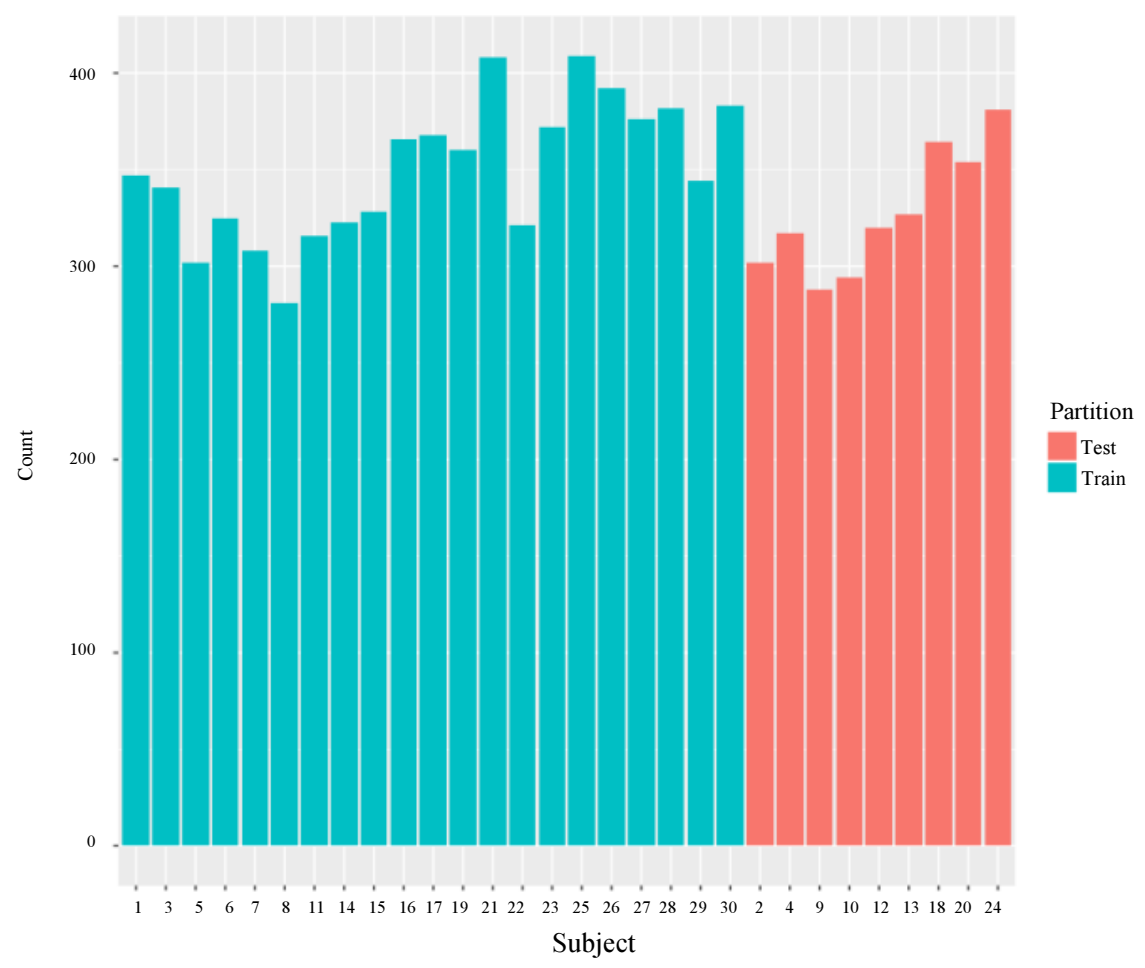

Fig. 3: Subject count visualization for train test partition

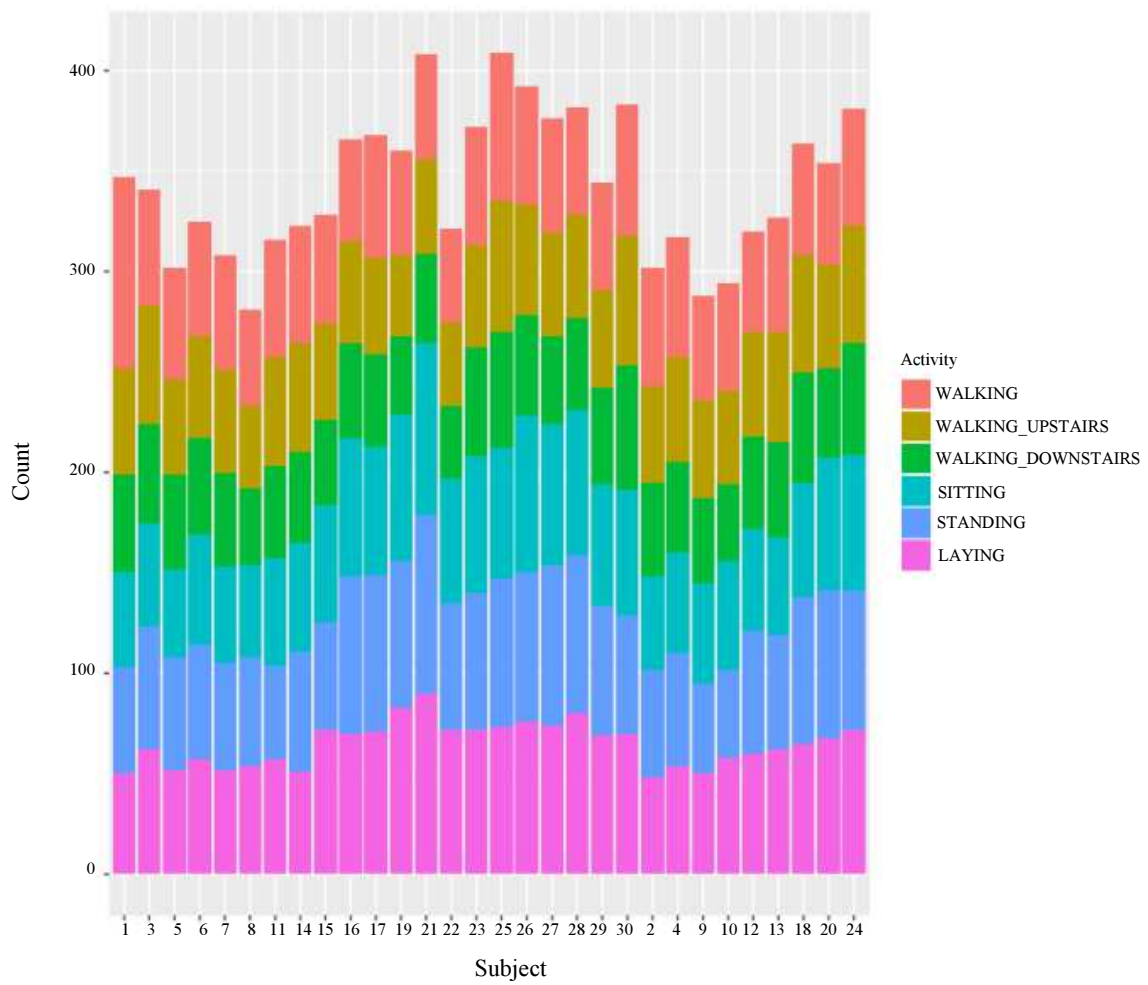

Fig. 4: Subject count visualization activities samples distribution 


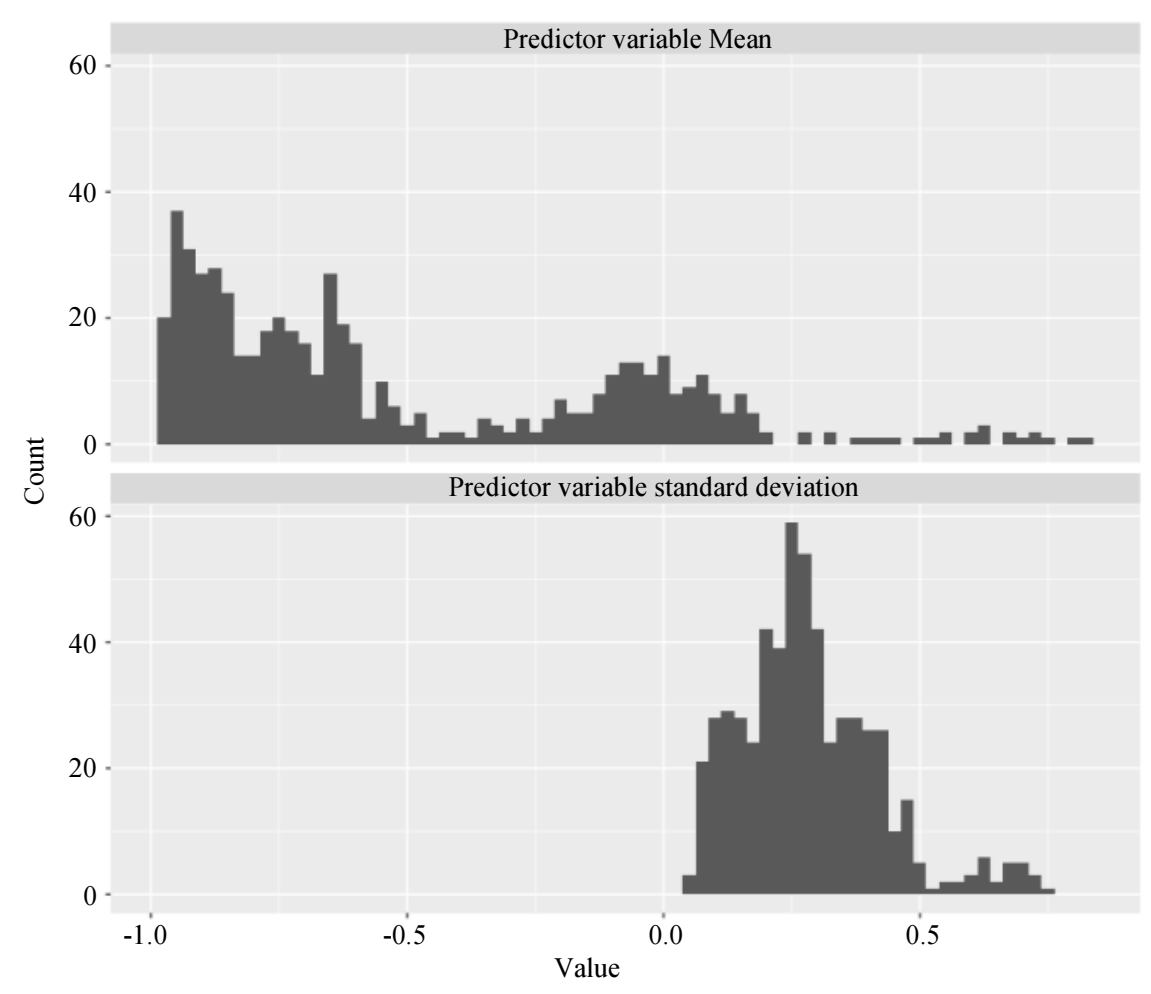

Fig. 5: Value vs count PVM-PVSD

\section{Obtained Results}

This section presents the experimental results obtained in the simulation procedure. The subject count visualization for train test partition and subject count visualization activities samples distribution graphs are shown as Fig. 3 and 4 respectively. Value vs count predictor variable mean and predictor variable standard deviation can be visualized through Fig. 5 .

Total number of samples: 7352; Out of total samples, 277 predictors realized. Seven fold cross-validated resampling is performed. Resampling results across tuning parameters are given in Table 1 .

The optimal value observed for this was mtry as equal to 10 . The confusion matrix obtained in this procedure is as follows:

\section{Reference}

\begin{tabular}{lllllll}
\hline Prediction & I & II & III & IV & V & VI \\
\hline I & 15.0 & 0.1 & 0.1 & 0.0 & 0.0 & 0.0 \\
II & 1.3 & 14.0 & 1.2 & 0.0 & 0.0 & 0.0 \\
III & 0.4 & 0.5 & 12.1 & 0.0 & 0.0 & 0.0 \\
IV & 0.0 & 0.0 & 0.0 & 15.5 & 1.5 & 0.0 \\
V & 0.0 & 0.0 & 0.0 & 1.6 & 17.2 & 0.0 \\
VI & 0.0 & 0.0 & 0.0 & 0.3 & 0.0 & 19.1 \\
\hline
\end{tabular}

Note: Entries in the confusion matrix are percentual average cell counts across resamples. Denotions are as follows: I - WALKING

\section{II - WALKING UPSTAIRS \\ III - WALKING DOWNSTAIRS \\ IV - SITTING \\ $\mathrm{V}$ - STANDING \\ VI - LAYING}

Generalization performance predictive analysis on the model: Confusion Matrix and Statistics:

Reference

\begin{tabular}{lllllll}
\hline Prediction & I & II & III & IV & V & VI \\
\hline I & 492 & 11 & 6 & 0 & 0 & 0 \\
II & 0 & 454 & 42 & 1 & 0 & 0 \\
III & 4 & 6 & 372 & 0 & 0 & 0 \\
IV & 0 & 0 & 0 & 433 & 15 & 0 \\
V & 0 & 0 & 0 & 56 & 517 & 0 \\
VI & 0 & 0 & 0 & 1 & 0 & 537 \\
\hline
\end{tabular}

The Overall Statistical performance measures are given in Table 2.

\section{Comparisons}

To compare the experimental analysis results of proposed procedure with some other approaches, we performed the experimental simulations on k- Nearest Neighbors approach and serialized implementation scenario approach also by keeping the simulation environment and system setup parameters same. 
Table 1: Resampling results across tuning parameters

\begin{tabular}{lll}
\hline Mtry & Accuracy $(\%)$ & Kappa \\
\hline 2 & 91.05 & 0.8921086 \\
5 & 92.71 & 0.9122211 \\
10 & 92.88 & 0.9141794 \\
15 & 92.30 & 0.9071583 \\
20 & 91.44 & 0.8968782 \\
\hline
\end{tabular}

Table 2: Overall Statistical performance

\begin{tabular}{ll}
\hline Parameters & Value \\
\hline Accuracy & $95.19 \%$ \\
$95 \%$ CI & $(0.9435,0.9593)$ \\
No Information Rate & 0.1822 \\
P-Value & $<2.2 \mathrm{e}-16$ \\
Kappa & 0.9421 \\
Exec time & $8568 \mathrm{Sec}$ \\
\hline
\end{tabular}

Table 3: Comparative analysis

\begin{tabular}{llll}
\hline & $\begin{array}{l}\text { k-Nearest } \\
\text { Neighbor }\end{array}$ & $\begin{array}{l}\text { Serialized } \\
\text { approach } \\
\text { scenario }\end{array}$ & $\begin{array}{l}\text { Adopted } \\
\text { procedure }\end{array}$ \\
\hline Accuracy & $86.31 \%$ & $92.7 \%$ & $95.19 \%$ \\
Kappa & 0.832 & 0.909 & 0.9421 \\
Exec time & $358.8 \mathrm{sec}$. & $40,320 \mathrm{sec}$. & $8,568 \mathrm{sec}$. \\
\hline
\end{tabular}

The comparative analysis is given in Table 3 . It can be observed that our adopted procedure outperforms with comparatively better accuracy and Kappa measure. It also gained speed up as $=\frac{40320}{8568} \simeq 4.7 x$

\section{Conclusion}

Human activity recognition (HAR) is a crucial and significant research domain in pattern recognition and pervasive computing. This paper surveys some recently developed significant approaches and their results in this domain. Consecutively, we presented our adopted procedure for human activities prediction and analysis. The model is applied for the experimental analysis of HAR Smartphone dataset. To tackle several grand challenges in this domain and developing more feasible solutions/approaches that can be practically more applicable in real scenarios, will be our future research possibility.

\section{Acknowledgment}

We would like to acknowledge Mr. Ajeet Singh and Ms. Neha for their assistance execution, editing and proofreading the paper.

\section{Author's Contributions}

Budi Padmaja: Made substantial contributions in formulating the concept, design and carried out experimentation, analysis and interpretation of data.
Venkata Rama Prasad Vaddella: Participated in concept formulation and statistical analysis.

Kota Venkata Naga Sunitha: Participated in design and helped in literature survey and drafting the manuscript.

All authors read and approved the final manuscript.

\section{Ethics}

This article is original and contains unpublished material. The corresponding author confirms that all of the other authors have read and approved the manuscript and no ethical issues involved.

\section{References}

Almaslukh, B., J. AlMuhtadi and A. Artoli, 2017. An effective deep autoencoder approach for online smartphone-based human activity recognition. Int. J. Comput. Sci. Netw. Security 17: 160-165.

Anguita, D., A. Ghio, L. Oneto, X. Parra and J.L. ReyesOrtiz, 2012. Human Activity Recognition on Smartphones Using a Multiclass Hardware-Friendly Support Vector Machine. Proceedings of the 4th International Conference on Ambient Assisted Living and Home Care, Dec. 03-05, Springer, Vitoria-Gasteiz, Spain, pp: 216-223. DOI: $10.1007 / 978-3-642-35395-6 \_30$

Anguita, D., A. Ghio, L. Oneto, X. Parra and J.L. ReyesOrtiz, 2013. A public domain dataset for human activity recognition using smartphones. Proceedings of the 21th International European Symposium on Artificial Neural Networks, Computational Intelligence and Machine Learning, Apr. 26-24, Bruges, Belgium, pp: 437-442.

Bhattacharya, S. and N.D. Lane, 2016. From smart to deep: Robust activity recognition on smartwatches using deep learning. Proceedings of the IEEE International Conference on Pervasive Computing and Communication Workshops, Mar. 14-18, IEEE Xplore Press, Sydney, NSW, Australia, pp: 1-6. DOI: 10.1109/PERCOMW.2016.7457169

Chen, Y., K. Zhong, J. Zhang, Q. Sun and X. Zhao, 2016. LSTM networks for mobile human activity recognition. Proceedings of the International Conference on Artificial Intelligence: Technologies and Applications, (ITA' 16), pp: 5053.

Cheng, W.Y. and A.E.A. Scotland, 2017. Human activity recognition from sensorbased large-scale continuous monitoring of parkinsons disease patients. Proceedings of the IEEE/ACM International Conference on Connected Health: Applications, Systems and Engineering Technologies, Jul. 17-19, IEEE Xplore Press, Philadelphia, PA, USA, pp: 249-250.

DOI: 10.1109/CHASE.2017.87 
Edel, M. and E. Kppe, 2016. Binarized-BLSTM-RNN based human activity recognition. Proceedings of the International Conference on Indoor Positioning and Indoor Navigation, Oct. 4-7, IEEE Xplore Press, Alcala de Henares, Spain, pp: 1-7. DOI: 10.1109/IPIN.2016.7743581

Gjoreski, H., J. Bizjak, M. Gjoreski and M. Gams, 2016. Comparing deep and classical machine learning methods for human activity recognition using wrist accelerometer. Proceedings of the Workshop on Deep Learning for Artificial Intelligence, (LAI' 16).

Ha, S. and S. Choi, 2016. Convolutional neural networks for human activity recognition using multiple accelerometer and gyroscope sensors. Proceedings of the International Joint Conference on Neural Networks, Jul. 24-29, IEEE Xplore Press, Vancouver, BC, Canada, pp: 381-388. DOI: 10.1109/IJCNN.2016.7727224

Ha, S., J.M. Yun and S. Choi, 2015. Multimodal convolutional neural networks for activity recognition. Proceedings of the IEEE International Conference on Systems, Man and Cybernetics, Oct. 9-12, IEEE Xplore Press, Kowloon, China, pp: 3017-3022. DOI: $10.1109 /$ SMC.2015.525

Hannink, J., T. Kautz, C.F. Pasluosta, K.G. Gaßmann and J. Klucken et al., 2017. Sensor-based gait parameter extraction with deep convolutional neural networks. IEEE J. Biomed. Health Inform., 21: 85-93. 10.1109/JBHI.2016.2636456

https://archive.ics.uci.edu/ml/datasets/human+activity+re cognition+using+smartphones

$\mathrm{Hu}, \mathrm{L}$., Y. Chen, S. Wang, J. Wang and J. Shen et al., 2016. Less annotation on personalized activity recognition using context data. Proceedings of the International IEEE Conferences on Ubiquitous Intelligence and Computing, Advanced and Trusted Computing, Scalable Computing and Communications, Cloud and Big Data Computing, Internet of People and Smart World Congress, Jul. 18-21, IEEE Xplore Press, Toulouse, France, pp: 327-332. DOI: 10.1109/UIC-ATC-ScalComCBDCom-IoP-SmartWorld.2016.0066
Padmaja, B., V.V. Rama Prasad and K.V.N. Sunitha, 2016a. Use of reality mining dataset for human behavior analysis: A survey. Proceedings of the International Conference on Information Systems Engineering, Apr. 20-22, IEEE Xplore Press, Los Angeles, CA, USA, pp: 38-42. DOI: 10.1109/ICISE.2016.16

Padmaja, B., V.V.R. Prasad and K.V.N. Sunitha, 2016b. TreeNet analysis of human stress behavior using socio-mobile data. J. Big Data, 3: 24-24. DOI: $10.1186 / \mathrm{s} 40537-016-0054-3$

Padmaja, B., V.V.R. Prasad and K.V.N. Sunitha, 2016c. Connecting productivity with social capital via daily mobile phone logs. Soc. Network., 5: 62-74.

Padmaja, B., V.V.R. Prasad and K.V.N. Sunitha, 2018a. A machine learning approach for stress detection using a wireless physical activity tracker. Int. J. Mach. Learn. Comput., 8: 33-38.

Padmaja, B., V.V. Rama Prasad, K.V.N. Sunitha and G. Vineeth Reddy, 2018b. Deep RNN based human activity recognition using LSTM architecture on smart phone sensor data. J. Fundamental Applied Sci.

Padmaja, B., P.V. Narasimha Rao, Y. Mohana Roopa and E. Krishna Rao Patro, 2018c. IoT based surveillance of agriculture and smart detection of infestation. J. Adv. Res. Dynamical Control System.

Sunitha, K.V.N., B. Padmaja and V.V. Rama Prasad, 2018. DetectStress: A novel stress detection system based on smartphone and wireless physical activity tracker. Proceedings of the 1st International Conference on Artificial Intelligence and Cognitive Computing, (ICC' 18), Springer, Singapore, pp: 67-80. DOI: 10.1007/978-981-13-1580-0_7

Wang, J., Y. Chen, S. Hao, X. Peng and L. Hu, 2017. Deep learning for sensor-based activity recognition: A survey. arXiv:1707.03502. 